\title{
The Role of miR-1 in the Heart: From Cardiac Morphogenesis to Physiological Function
}

\author{
Fangfei Deng ${ }^{1-3}$, Xinran $X \mathbf{u}^{1}$ and Yi-Han Chen ${ }^{1-4 *}$ \\ ${ }^{1}$ Key Laboratory of Basic Research in Cardiology of the Ministry of Education of China, Tongji University, Shanghai 200120, China \\ ${ }^{2}$ Institute of Medical Genetics, Tongji University, Shanghai 200092, China \\ ${ }^{3}$ Department of Cardiology, East Hospital, Tongji University, Shanghai 200120, China \\ ${ }^{4}$ Department of Pathology and Pathophysiology, Tongji University School of Medicine, Shanghai 200092, China
}

\begin{abstract}
Heart is pumping throughout life of animals ever since embryogenesis. The mammalian heart consists of four chambers along with specialized myocardial system for electrical conduction and force production, which guarantees the heart to pump efficiently. miR-1, the cardiac specific mi RNA, is suggested to play essential roles in cardiogenesis and in regulating physiological function of heart. During early stage of cardio genesis, miR-1 promotes cardiac mesoderm induction. In the subsequent morphogenesis, miR-1 controls cell fate of various lineages and the balance between proliferation and differentiation so that cardiac chambers could develop normally. In postnatal life, miR-1 modulates atrioventricular and ventricular conduction at multiple levels and contributes to the formation of organized sarcomere. Advance in the role miR-1 plays in cardiac biological processes have put new perspectives on mechanisms of heart diseases.
\end{abstract}

Keywords: MicroRNA-1; Cardiogenesis; Electrophysiology

\section{Introduction}

The heart which acquires its delicate structure from embryonic development pumps uninterruptedly throughout the life of all animals. Morphogenesis as well as maintenance of cardiac function requires precise regulation of various gene expressions. During cardiogenesis, signaling pathways and transcription factors exert spatiotemporal control of downstream gene transcription to guarantees cardiac morphogenesis progresses properly. In postnatal period, this regulatory network which is further joined by large quantity of enzymes and other participant's maintains rhythmic contraction. Some regulators in this network are effective in specific stage of life. While others, like GATA4, have shown to be essential both for embryonic development and postnatal heart function [1,2]. Recently, a novel regulatory mechanism involving post-transcriptional regulation by microRNAs (miRNAs) have been shown to function in both life stages [3].

MiRNAs are classes of small, non-coding RNAs that regulate target mRNAs by interacting with their 3'UTR in a sequence-specific manner [4]. Advances in miRNA analysis have made it possible to identify the role of miRNAs in heart. Disruption of Dicer allele, which is essential for processing of pre-miRNAs into mature form, in early stage of cardiogenesis results in profound heart defects, and the embryos die from cardiac failure by E12.5 [5]. Cardiac-specific deletion of Dicer allele in later stage of development causes enlargement of chambers and myocyte hypertrophy that leads to functional defects resemble dilated cardiomyopathy [6,7]. These studies indicate that modifications in miRNA biogenesis affect both embryonic and adult myocardial morphology and function [6].

MiR-1 is most abundantly and specifically expressed in heart [5]. Expression of miR-1 can be detected in developing mouse heart as early as E8.5, and the expression becomes even more robust after birth [8]. MiR-1 null mice manifest morphological, electrophysiological and functional defects. mRNA expression microarray analysis of mutant and wide-type hearts reveals dysregulation of numerous cardiac transcription factor genes. These genes cluster into several categories, including regulators of cell cycle, cardiac differentiation and conductive system [5]. Here we review the role miR-1 plays in morphogenesis during cardiac development and in regulating physiological function in postnatal period.

\section{MiR-1 Modulates Cell Fate Decision and Controls Chamber Development during Cardiogenesis}

Cell fate decision refers to the process that pluripotent progenitors or stem cells adopt characteristics which belong to a specific lineage while suppress the potential of other lineages [9]. MiR-1 is suggested to regulate lineage-specific determination both in embryonic stem cell (ESC) and cardiac progenitors. Over expression of miR-1 in mouse ESC promotes mesodermal induction and early cardiac markers expression. On the other hand, down regulation of miR-1 in mESCs shows delayed differentiation into mesodermal progenitors. MiR-1 also represses a number of nonmusical gene expressions, i.e. endoderm markers Afp and Hnf4a as well as neuron marker Ncam1 [9]. These data indicate that miR-1 plays a major role in promoting the progression of mesodermal progenitors by up regulating cardiac mesodermal gene expression while down regulating genes belong to other cell lineages. Further analysis identifies that the Notch ligand Delta 1 (Dll1) is a direct target of miR-1, and the Dll1 knockdown mESCs show greater propensity toward cardiac cell fate and form beating cardiomyocyte earlier than wide type [9].

MiR-1's role in determination of cardiac progenitors has also been explored by in vivo study. MiR-1 deleted Drosophila shows

*Corresponding author: Yi-Han Chen, Key Laboratory of Basic Research in Cardiology of the Ministry of Education of China, Tongji University, No.150 Jimo Road, Shanghai 200120, China, Tel: +086-21-58766224; Fax: +086-21-65989086; E-mail: yihanchen@tongji.edu.cn

Received January 02, 2014; Accepted January 26, 2014; Published January 31 2014

Citation: Deng F, Xu X, Chen YH (2014) The Role of miR-1 in the Heart: From Cardiac Morphogenesis to Physiological Function. Human Genet Embryol 4: 119. doi:10.4172/2161-0436.1000119

Copyright: (C) 2014 Deng F, et al. This is an open-access article distributed unde the terms of the Creative Commons Attribution License, which permits unrestricted use, distribution, and reproduction in any medium, provided the original author and source are credited. 
overabundant progenitor pool and decreased cardioblasts. The Eve expressing progenitor cells undergo asymmetric division and give rise to two distinct lineages of daughter cells: one is pericardial cell, the other is muscle cell. However the $\mathrm{Eve}^{+}$pericardial cell is missing in the miR-1 mutants [10]. This experiment suggests miR-1 expression is required for development of the pericardial cell lineage, and its absence leads to the missing of entire lineage. In vivo evidence also indicates Dll1 as a target of miR-1 [10].

Notch pathway mediates cell fate decision by asymmetric expression of Notch receptor and Notch ligand Delta between opposing cells [11], which eventually guide the distinct biochemical events within the cells [12]. The function of Notch pathway in cardiogenesis has also been studied extensively. Nemir and colleagues 'research on ESCs supports the idea that inhibition of Notch signaling is essential for mesodermal induction of ESC, and eventually myocardial cell fate [13]. An in vivo study also shows Notch signaling regulates cardiac progenitor cell fate by modulating balance between muscle and non-myogenic cell lineages [14].

Cardiogenesis is exceptionally sensitive to the dose of Notch and $D l l$. So the quantity of ligand and receptor present on the surface of cells is a tightly regulated parameter in the Notch pathway [12]. As a novel regulator of cell fate decision, miR-1 repression of Dll 1 and "fine tune" the signal exchange in Notch pathway is likely one of the major mechanisms. In ESCs, miR-1 down regulation of Dll1 might account for inhibition of Notch1 signaling in the process of myocardial progression. Nonetheless questions like how expression of miR-1 is induced and how ligand and receptor interact with each other in this process need to be further explored. In cardiac progenitors undergo asymmetric cell division, miR-1 seems to suppress Dll1 and helps maintain active Notch receptor in signaling receiving cells. And the Notch receptor regulates specific gene expression thus dictates cell lineage. In order to specify the detailed mechanism of this process, it is important to examine miR-1's function in asymmetric division.

What worth mention is that miR-1 regulates cardiac cell fate by directing differentiation as opposed to proliferation in Drosophila [10]. So it will be interesting to determine whether it exerts similar function in mammals. To accommodate an advanced circulation system, mammals have adopted hearts with four chambers and the development which is a key event in the building plan of mammalian heart. Formation of the cardiac chambers displays simultaneous proliferation and differentiation. The proliferation and differentiation of chamber myocardium show highly-localized regulation which requires expansion of the complexity of regulatory network in Drosophila. Developing chambers manifest trans mural pattern of growth, with the highest proliferation rate in compact layer as well as progressive pattern of differentiation. This regional growth and differentiation of chamber myocardium ensues the "ballooning" model of chamber formation [3]. There are studies show miR-1 participates in the regulation of chamber expansion and differentiation. The miR-1 transgenic embryos show thin myocardium compact layer with decreased cycling myocardium. This is due to premature differentiation, early withdrawal from cell cycle, proliferation defect and failure of ventricular cardio myocyte expansion caused by excessive miR1 [8]. Conversely, miR-1-2 deletion results in cardiac hypertrophy and ventricular septum defect (VSD). Gene expression analysis of mutants shows up regulation of cardiogenic transcriptional factors compared with wide type [5]. According to what mentioned above, miR-1 mediates cell cycle arrest and differentiation of cardiomyocyte during chamber development. The study also suggests cardiac expression of miR-1 is serum response factor (SRF)-dependent [8].
SRF has long been acknowledged as a critical regulator for chamber differentiation and functional maturation [15]. Cardiac conditional knockout (CKO) of SRF results in morphological and functional defects, presented as reduced thickness of a trial and ventricular compact layer, poor trabeculation and losing rhythmic heart beating, pericardial effusion which are due to disrupted sarcomere organization. The atrophic heart does not display significant change in proliferation rate, yet shows a impaired expression of cardiac specific genes $[16,17]$. The cardiac chamber differentiation process shows inverse relationship between proliferation and differentiation. SRF is not considered as a inhibitory transfactor for cell cycle, rather it exert gene silencing activity through miRNAs [3]. miR-1 mediates cell cycle withdrawal and directly targets Hand2 [8].

Hand factors are recognized as important regulators for ventricular cardiomyocyte proliferation, differentiation, morphogenesis and conduction [18]. Homozygous Hand2 null mouse displays a single leftsided ventricular chamber and aberrant connection of outflow tract that is lethal between E9,5-E10. Molecular analysis reveals impaired expression of cardiogenic transcription factors without influencing cardiomyocyte differentiation markers in mutants. Hand2 is crucial for chamber morphogenesis and development of cardiac neural crestderived tissues [19], However overdose of Hand2 is also associated with heart abnormalities [20]. It is likely that miR-1 exert its negative regulation of myocyte proliferation partly via Hand2 during chamber differentiation [21]. However chamber formation is a local process, whether miR-1 expresses in a region-specific pattern, if so how it is regulated remains to be elucidated. However, it is safe to say that miR1 induced by SRF helps refining transcription activity and modulates chamber development at least partially via targeting Hand2.

\section{MiR-1 Regulates Electrical and Contractile Activity of the Heart}

Efficient contraction of heart is the result of highly organized conductive system and effective electromechanical coupling. Electrical stimulation proceeds from apex toward the base in ventricles and repolarize in reverse order [22]. Spatial heterogeneous action-potential duration and conductance guarantee depolarization and repolarization propagate orderly [23]. To ensure the heterogeneity, heart has adopted a spatial specific transcriptional network that tightly regulates expression of ion channel and gap junction. When stimuli reach working cardiomyocyte, it triggers release of calcium which binds to troponin $\mathrm{C}$ followed by myosin-actin cross-bridge formation. In this process, myosin light chain kinase (MLCK) potentiates the force and rate of cross-bridge recruitment in cardiomyocyte and may serve as a major target in regulation of cardiac contraction [24].

Perturbation the dose of miR-1 results in electrophysiological and contractile defects. The miR-1 homozygous null mice show prolonged QRS complex and prolonged PR and QT intervalson Surface electrocardiograph, indicating defects in atrioventricular (AV) and ventricular conduction. Echocardiography also reveals severely impaired fractional shortening with poor systolic function [25]. MiR-1 over expression mice develops frequent atrioventricular block of varying degree as evidenced by prolonged PR interval [26]. The transgenic mice also show impaired contractile and diastolic function that might due to damaged sarcomere assembly [27].

The miR-1 deletion mice show elevated Irx 4 and down regulated Irx 5 which is a target of miR-1-2 [5]. Three members of the Iroquois homeobox (Irx) family, Irx3, Irx4 and Irx 5 have been proved to play individual as well as cooperative and antagonistic roles in regulation 
of electrophysiology. Irx3 establishes fast conduction in His-purkinje network [28]. Irx5 builds repolarization gradient of mouse ventricle [23]. Irx3; Irx 5 double knockout (DKO) mice show AV and ventricular conduction defects, indicating a cooperative role of both in the modulation of proximal ventricular conduction. There is also an antagonistic mechanism between Irx 5 and Irx 3 in the regulation of ventricular depolarization [22]. Cooperation of Irxs happens between Irx4 and Irx5 in control of Kcnd2 promoter activation [29]. So precise dose of individual Irxs as well as relative ratio of Irxs is crucial for establishment of proper conduction. By directly targeting Irx5, miR-1 may modulate relative ratio between Irxs to regulate $\mathrm{AV}$ and ventricular conduction. How miR-1 influence expression of other Irxs still need to be further addressed.

miR-1 also regulates gap junction-forming and iron channel proteins directly [30,31]. miR-1 slows ventricular conduction through directly down regulation of $\mathrm{Cx} 43$ which expresses in working myocardium and facilitates coupling of the high speed conduction pathway to the surrounding myocytes [32]. MiR-1 also targets the cardiac L-type calcium channel gene CACNA1C (CAV1.2), the main calcium channel in heart [31]. SomiR-1 might control electrophysiology through targeting multiple levels. But how miR-1 orchestrates different levels of regulation needs to be further explored. In the miR-1 up-/ down-regulated mice that manifest abnormal contractile function, two sets of genes are dysregulated. For miR-1 over expression mice, cardiac MLCK which functions to maintain cardiac contraction and sarcomeric assembly is significantly decreased. This is accompanied by decrease of calmuldin which is also critical for sarcomere assembly and function [27]. As for the miR-1 null mutants, a MLCK isoform, Telokin, which is supposed to specifically express in smooth muscle aberrantly upregulates in cardiomyocytes [25]. Both increased Telokin and decreased cardiac MLCK impair phosphorylation of MLC2 that results in defected assembly of the myosin thick filaments. Another study also indicates miR-1 plays important role in regulating contractility of cardiomyocyte by controlling calcium homeostasis [33]. MiR-1 mediates expression of different MLCK is forms and controls calcium homeostasis to maintain forceful contraction.

\section{Concluding Remarks}

Studies on miR-1's function in heart have uncovered novel mechanisms underlying broad spectrum of cardiac diseases. Although miR-1 has not been related to congenital heart deformities, there is ever increasing knowledge of miR-1' role in arrhythmia and cardiac dysfunction. Expression of miR-1 is lost in myotonic dystrophy patients, concomitant with up regulation of its targets $\mathrm{Cx} 43$ and CAV1.2 which may account for arrhythmia occurred in these patients [31]. Besides, its expression is significantly reduced in chronic a trial fibrillation patients [34], although the implication has not been fully understood. miR-1 may also play a role in ischemic arrhythmia in patients with coronary heart disease [30]. In samples from end-stage heart failure patients and hypertrophic cardiomyopathy mouse model, miR-1 is significantly down regulated $[33,35]$. There is also study shows miR-1 is involved in the development of diabetic cardiac dysfunction in mouse model [36].

MiR-1 is an example that a single miRNA plays critical roles in cardiogenesis as well as maintaining normal cardiac function. However, the target genes identified thus far are only a small fraction of the total genes directly respond to miR-1 in the heart. The application of genomewide enhancer/repressor screen has aided in uncovering multiple factors which functions in common pathways targeted by miR-1 and other miRNAs [37]. With increasing knowledge about functions of miRNAs in heart, we are expecting to see a regulatory network consists of interrelated miRNAs that intertwine with well-described signaling and transcription factor networks to determine the development and pathologenesis of the heart [21].

\section{Acknowledgements}

This work was supported by the Major International Joint Research Program Fund of China (81120108004, to Yi-Han Chen), the Foundation for Innovative Research Groups of China (81221001, to Yi-Han Chen), the National Key Basic Research Program of China (2013CB531100, to Yi-Han Chen), the National Natural Science Foundation of China (1271214, to Yi-Han Chen), the Shanghai Natural Science Fund (10JC1414700, to Yi-Han Chen), the Fundamental Research Funds for the Central Universities $(2011 \mathrm{KJ} 005$, to Xinran Xu), the Doctoral Program Foundation of Ministry of Education of China (20120072120009, to Xinran Xu), and the Training Program for Outstanding Young Medical Talents in Pudong District (PWRq2011-05, to Xinran Xu).

\section{References}

1. Kuo CT, Morrisey EE, Anandappa R, Sigrist K, Lu MM, et al. (1997) GATA4 transcription factor is required for ventral morphogenesis and heart tube formation. Genes Dev 11: 1048-1060.

2. Wang J, Paradis P, Aries A, Komati H, Lefebvre C, et al. (2005) Convergence of protein kinase $C$ and JAK-STAT signaling on transcription factor GATA-4. Mol Cell Biol 25: 9829-9844.

3. Rosenthal N, Harvey RP (2010) Heart development and regeneration. (1stedn) Elsevier Inc. London, UK.

4. Small EM, Olson EN (2011) Pervasive roles of microRNAs in cardiovascular biology. Nature 469: 336-342.

5. Zhao Y, Ransom JF, LiA, Vedantham V, von Drehle M, et al. (2007) Dysregulation of cardiogenesis, cardiac conduction, and cell cycle in mice lacking miRNA-1-2. Cell 129: 303-317

6. da Costa Martins PA, Bourajiaj M, Gladka M, Kortland M, van Oort RJ, et al. (2008) Conditional dicer gene deletion in the postnatal myocardium provokes spontaneous cardiac remodeling. Circulation 118: 1567-1576.

7. Chen JF, Murchison EP, Tang R, Callis TE, Tatsuguchi M, et al. (2008) Targeted deletion of Dicer in the heart leads to dilated cardiomyopathy and heart failure. Proc Natl Acad Sci U S A 105: 2111-2116.

8. Zhao Y, Samal E, Srivastava D (2005) Serum response factor regulates a muscle-specific microRNA that targets Hand2 during cardiogenesis. Nature 436: $214-220$

9. Ivey KN, Muth A, Arnold J, King FW, Yeh RF, et al. (2008) MicroRNA regulation of cell lineages in mouse and human embryonic stem cells. Cell Stem Cell 2 : 219-229.

10. Kwon C, Han Z, Olson EN, Srivastava D (2005) MicroRNA1 influences cardiac differentiation in Drosophila and regulates Notch signaling. Proc Natl Acad Sci U S A 102: 18986-18991.

11. Jan YN, Jan LY (1998) Asymmetric cell division. Nature 392: 775-778.

12. Artavanis-Tsakonas S, Rand MD, Lake RJ (1999) Notch signaling: cell fate control and signal integration in development. Science 284: 770-776.

13. Nemir M, Croquelois A, Pedrazzini T, Radtke F (2006) Induction of cardiogenesis in embryonic stem cells via downregulation of Notch1 signaling. Circ Res 98 1471-1478.

14. Han Z, Bodmer R (2003) Myogenic cells fates are antagonized by Notch only in asymmetric lineages of the Drosophila heart, with or without cell division. Development 130: 3039-3051.

15. Miano JM, Long X, Fujiwara K (2007) Serum response factor: master regulator of the actin cytoskeleton and contractile apparatus. Am J Physiol Cell Physio 292: C70-81.

16. Parlakian A, Tuil D, Hamard G, Tavernier G, Hentzen D, et al. (2004) Targeted inactivation of serum response factor in the developing heart results in myocardial defects and embryonic lethality. Mol Cell Biol 24: 5281-5289.

17. Niu Z, Yu W, Zhang SX, Barron M, Belaguli NS, et al. (2005) Conditional mutagenesis of the murine serum response factor gene blocks cardiogenesis and the transcription of downstream gene targets. J Biol Chem 280: 3253132538.

18. Vincentz JW, Barnes RM, Firulli AB (2011) Hand factors as regulators of cardiac 
Citation: Deng F, Xu X, Chen YH (2014) The Role of miR-1 in the Heart: From Cardiac Morphogenesis to Physiological Function. Human Genet Embryol 4: 119. doi:10.4172/2161-0436.1000119

morphogenesis and implications for congenital heart defects. Birth Defects Res A Clin Mol Teratol 91: 485-494.

19. Srivastava D, Thomas T, Lin Q, Kirby ML, Brown D, et al. (1997) Regulation of cardiac mesodermal and neural crest development by the bHLH transcription factor, dHAND. Nat Genet 16: 154-160.

20. Tamura M, Hosoya M, Fujita M, lida T, Amano T, et al. (2013) Overdosage of Hand2 causes limb and heart defects in the human chromosomal disorder partial trisomy distal 4q. Hum Mol Genet 22: 2471-2481.

21. Liu N, Olson EN (2010) MicroRNA regulatory networks in cardiovascular development. Dev Cell 18: 510-525.

22. Gaborit N, Sakuma R, Wylie JN, Kim KH, Zhang SS, et al. (2012) Cooperative and antagonistic roles for Irx3 and Irx5 in cardiac morphogenesis and postnatal physiology. Development 139: 4007-4019.

23. Costantini DL, Arruda EP, Agarwal P, Kim KH, Zhu Y, et al. (2005) The homeodomain transcription factor Irx5 establishes the mouse cardiac ventricular repolarization gradient. Cell 123: 347-358

24. Chan JY, Takeda M, Briggs LE, Graham ML, Lu JT, et al. (2008) Identification of cardiac-specific myosin light chain kinase. Circ Res 102: 571-580.

25. Heidersbach A, Saxby C, Carver-Moore K, Huang Y, Ang YS, et al. (2013) microRNA-1 regulates sarcomere formation and suppresses smooth muscle gene expression in the mammalian heart. Elife 2: e01323.

26. Zhang Y, Sun L, Zhang Y, Liang H, Li X, et al. (2013) Overexpression of microRNA-1 causes atrioventricular block in rodents. Int J Biol Sci 9: 455-462.

27. Ai J, Zhang R, Gao X, Niu HF, Wang N, et al. (2012) Overexpression of microRNA-1 impairs cardiac contractile function by damaging sarcomere assembly. Cardiovasc Res 95: 385-393.

28. Zhang SS, Kim KH, Rosen A, Smyth JW, Sakuma R, et al. (2011) Iroquois homeobox gene 3 establishes fast conduction in the cardiac His-Purkinje network. Proc Natl Acad Sci U S A 108: 13576-13581.
29. He W, Jia $Y$, Takimoto $K$ (2009) Interaction between transcription factors Iroquois proteins 4 and 5 controls cardiac potassium channel Kv4.2 gene transcription. Cardiovasc Res 81: 64-71.

30. Yang B, Lin H, Xiao J, Lu Y, Luo X, et al. (2007) The muscle-specific microRNA miR-1 regulates cardiac arrhythmogenic potential by targeting GJA1 and KCNJ2. Nat Med 13: 486-491.

31. Rau F, Freyermuth F, Fugier C, Villemin JP, Fischer MC, et al. (2011) Misregulation of miR-1 processing is associated with heart defects in myotonic dystrophy. Nat Struct Mol Biol 18: 840-845.

32. Lo CW (2000) Role of gap junctions in cardiac conduction and development: insights from the connexin knockout mice. Circ Res 87: 346-348.

33. Ali R, Huang Y, Maher SE, Kim RW, Giordano FJ, et al. (2012) miR-1 mediated suppression of Sorcin regulates myocardial contractility through modulation of Ca2+ signaling. J Mol Cell Cardiol 52: 1027-1037.

34. Stillitano F, Lonardo G, Giunti G, Del Lungo M, Coppini R, et al. (2013) Chronic atrial fibrillation alters the functional properties of If in the human atrium. $J$ Cardiovasc Electrophysiol 24: 1391-1400.

35. Bagnall RD, Tsoutsman T, Shephard RE, Ritchie W, Semsarian C (2012) Globa microRNA profiling of the mouse ventricles during development of severe hypertrophic cardiomyopathy and heart failure. PLoS One 7: e44744.

36. Yildirim SS, Akman D, Catalucci D, Turan B (2013) Relationship between downregulation of miRNAs and increase of oxidative stress in the development of diabetic cardiac dysfunction: junctin as a target protein of miR-1. Cel Biochem Biophys 67: 1397-1408.

37. King IN, Qian L, Liang J, Huang Y, Shieh JT, et al. (2011) A genome-wide screen reveals a role for microRNA- 1 in modulating cardiac cell polarity. Dev Cell 20: 497-510. 